\title{
Reduced expression of SRY-box containing gene 17 correlates with an unfavorable melanoma patient survival
}

\author{
JING LU ${ }^{1,2^{*}}$, GUOHONG ZHANG ${ }^{1,3 *}$, YABIN CHENG $^{1}$, YUN TANG $^{1}$, \\ ZIMING DONG ${ }^{2}$, KEVIN J. McELWEE ${ }^{1}$ and GANG LI ${ }^{1}$ \\ ${ }^{1}$ Department of Dermatology and Skin Science, Research Pavilion, Vancouver Coastal Health Research Institute, \\ University of British Columbia, Vancouver, BC V5Z 1L8, Canada; ${ }^{2}$ Department of Pathophysiology, \\ Basic Medical College, Zhengzhou University, Zhengzhou, Henan 450001; ${ }^{3}$ Department of \\ Pathology, Shantou University Medical College, Shantou, Guangdong 515041, P.R. China
}

Received May 30, 2014; Accepted July 31, 2014

DOI: $10.3892 /$ or.2014.3534

\begin{abstract}
SRY-box containing gene 17 (Sox17), a transcription factor, is considered as an antagonist to canonical $\mathrm{Wnt} / \beta$-catenin signaling in several types of malignant tumors. As the influence of Sox17 in the pathogenesis of human melanoma is still unknown, the investigation of Sox 17 expression in melanoma is warranted and its prognostic value is of great interest. In the present study, Sox17 expression was examined in 525 cases of melanocytic lesions (33 common acquired nevi, 59 dysplastic nevi, 291 primary melanomas and 142 metastatic melanomas) at different stages by tissue microarray. The correlation of Sox17 expression with melanoma progression and its prognostic value in melanoma patients were examined. We also analyzed the correlation between Sox17 and cyclindependent kinase inhibitor p27 expression in 374 melanoma samples. The results showed that Sox 17 expression was significantly decreased in primary and metastatic melanoma compared to common acquired nevi and dysplastic nevi $\left(\mathrm{P}=2.4 \times 10^{-17}\right)$. Furthermore, Sox 17 expression was inversely correlated with American Joint Committee on Cancer stage $\left(\mathrm{P}=4.6 \times 10^{-15}\right)$, thickness $(\mathrm{P}=0.00004)$ and ulceration $(\mathrm{P}=0.03)$. Notably, reduced Sox17 expression was correlated with a poorer overall and disease-specific 5- and 10-year survival of the patients. Multivariate Cox regression analyses indicated that Sox17 is an independent prognostic marker for melanoma patients. Moreover, we found a significant positive correlation between Sox17 and p27 expression in melanoma biopsies;
\end{abstract}

Correspondence to: Dr Jing Lu, Department of Pathophysiology, Basic Medical College, Zhengzhou University, No. 100 Science Road, Zhengzhou, Henan 450001, P.R. China

E-mail: lujing@zzu.edu.cn

Dr Kevin J. McElwee, Research Pavilion, 828 West 10th Avenue, Vancouver, BC V5Z 1L8, Canada

E-mail: kmcelwee@interchange.ubc.ca

*Contributed equally

Key words: Sox17, melanoma, prognosis, progression, p27 their concomitant expression was closely correlated with the survival of melanoma patients. Taken together, decreased Sox17 expression is correlated with melanoma progression, an unfavorable survival of melanoma patients and is an independent molecular prognostic factor for melanoma.

\section{Introduction}

Melanoma is the most deadly form of skin cancer arising from the melanocytes in the skin epidermis. It is characterized by rapid tumor progression, metastasis and a poor prognosis (1). Although melanoma accounts for only $4 \%$ of all dermatological cancers, it is responsible for $80 \%$ of deaths from skin cancer; less than $10 \%$ of patients with metastatic melanoma survive for 5 years $(1,2)$. The discovery and application of biomarkers, in conjunction with traditional cancer diagnosis, staging and prognosis, could be useful in improving early diagnosis, screening and subsequent management and treatment of melanoma patients (3-5). However, at present, reliable markers are still lacking and the prognosis of melanoma patients remains poor. Therefore, a better understanding of the regulating factors contributing to melanoma initiation, progression and metastasis is needed.

The Sox gene family was first identified by homology to the high mobility group (HMG) box of the sex-determining gene $S R Y(6)$. There are at least 30 members in the Sox family, which are expressed in various types of cells and tissues at different developmental stages (7). Sox17 encodes an HMG box transcription factor and has been demonstrated to play critical roles in the regulation of vascular development (8), differentiation of embryonic stem cells (9), hematopoietic development (10) and tumor progression $(11,12)$.

Accumulating evidence indicates that activation of Wnt/ $\beta$-catenin signaling is one of the direct causes of tumor development (13-15). The nuclear accumulation of $\beta$-catenin, a hallmark of Wnt activation, is particularly enhanced in the invasive front of tumors and metastasized colon cancer cells, suggesting that the activation of $\mathrm{Wnt} / \beta$-catenin signaling is important for colon cancer progression (16). Notably, some research has demonstrated that Sox17 inhibits the canonical Wnt/ $\beta$-catenin signaling in malignant tumors $(10,11,17)$. 
Recombinant Sox 17 not only promoted the level of the Wnt antagonist SFRP1, but also decreased the expression level of Wnt/Frizzled and endogenous $\beta$-catenin in HOG cells, leading to cell cycle exit and differentiation (10). On the other hand, Sox 17 expression was found to be downregulated in colon cancer cells and Sox17 overexpression inhibited colon cancer cell proliferation and reduced the efficiency of colony formation (18). Other research has illustrated that Sox 17 protects benign gastrointestinal tumors from malignant progression at an early stage of tumorigenesis and downregulation of Sox 17 contributes to malignant progression through promotion of Wnt activity (19). These results suggest that Sox 17 plays a tumor suppressor role in gastric and colorectal cancer development. However, to our knowledge, there is no report on the influence of Sox 17 involvement in the biological regulation of human melanoma. Here, we investigated Sox 17 expression in a large set of melanocytic lesions at different stages by tissue microarray, investigated the correlation of Sox 17 expression to melanoma progression and determined its prognostic value in patients with melanoma.

The cyclin-dependent kinase (CDK) inhibitor p27, also known as Kip1, is an atypical tumor suppressor that regulates $\mathrm{G}_{0}-\mathrm{S}$ phase transitions and plays important roles in cell proliferation, motility and apoptosis (20). Reduced nuclear p27 expression is associated with a poor prognosis in several types of cancers $(20,21)$. Our previous study also demonstrated that $\mathrm{p} 27$ expression is inversely associated with the survival of melanoma patients (22). Sox17-siRNA transfection of gastric cancer MKN45 cells led to observably increased expression of cyclin D1 while p27 expression was markedly decreased (12). Furthermore, p27 expression was found to be significantly induced in liver-specific $\beta$-catenin knockdown mice (23). Based on these findings, we further examined the correlation between Sox17 and p27 expression in melanoma biopsies and analyzed the combined effect of Sox17 and p27 expression in predicting patient outcome.

\section{Materials and methods}

Ethics statement. The use of human skin tissues in this study was approved by the Clinical Research Ethics Board of the University of British Columbia $(24,25)$. The study was conducted according to the principles expressed in the Declaration of Helsinki.

Tissue microarray (TMA) construction. The collection of melanoma patient specimens and the construction of the TMA have been previously described $(24,25)$. Briefly, formalin-fixed, paraffin-embedded tissues from 49 common acquire nevi, 100 dysplastic nevi, 402 primary melanomas and 162 metastatic melanomas were used for this TMA construction. All specimens were obtained from the 1990 to 2009 archives of the Department of Pathology, Vancouver General Hospital. The most representative tumor area was carefully selected and marked on the hematoxylin and eosin stained slides, and the TMAs were assembled using a tissuearray instrument (Beecher Instruments, Silver Spring, MD, USA). Due to loss of biopsy cores or insufficient tumor cells present in the cores, 33 common acquired nevi (CAN) which are prototypical benign melanocytic nevi, 59 dysplastic nevi,

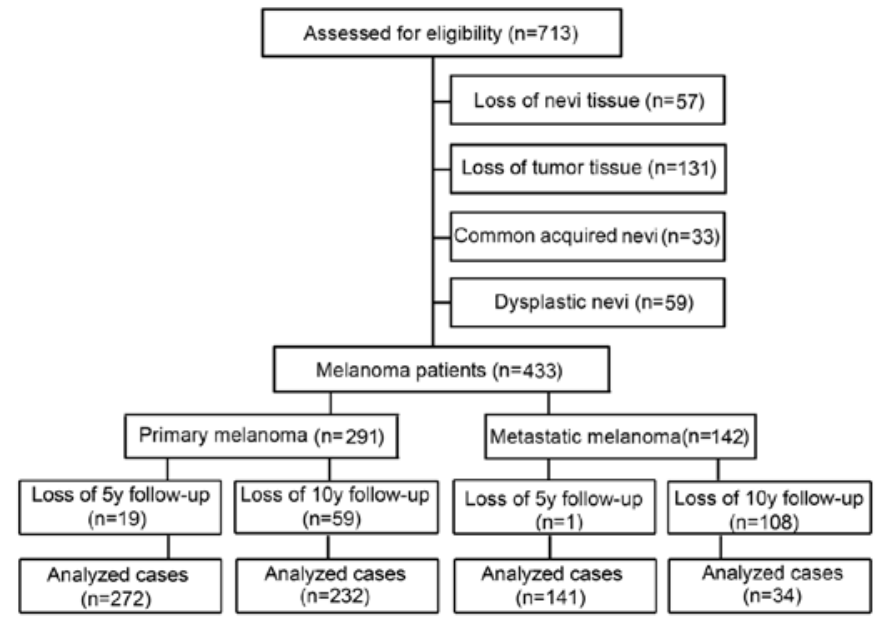

Figure 1. Diagram shows patient inclusion and exclusion.

291 primary melanomas, and 142 metastatic melanomas were able to be evaluated for Sox 17 staining (Fig. 1).

Immunohistochemistry of TMA. Immunohistochemistry was performed as described previously $(24,25)$. TMA slides were dewaxed at $55^{\circ} \mathrm{C}$ for $30 \mathrm{~min}$ and then washed with xylene. Tissues were rehydrated by a series of washes in 100,95 and $80 \%$ ethanol, followed by two washes in distilled water. Antigen retrieval was performed by heating the samples at $95^{\circ} \mathrm{C}$ for $30 \mathrm{~min}$ in $10 \mathrm{mmol} / \mathrm{l}$ sodium citrate $(\mathrm{pH} 6.0)$. After inactivating the endogenous peroxidase by incubation in $3 \%$ $\mathrm{H}_{2} \mathrm{O}_{2}$ for 30 min and blocking with universal blocking serum for $30 \mathrm{~min}$, the slides were incubated with a primary anti-Sox 17 antibody (1:100; EMD Millipore Corporation, Billerica, MA, USA) at $4^{\circ} \mathrm{C}$ overnight. Negative controls were constructed by omitting the Sox 17 antibody during the primary antibody incubation. The slides were then incubated with biotin-labeled secondary antibody and streptavidin-peroxidase for $30 \mathrm{~min}$ each, followed by developing with a diaminobenzidine substrate kit (DAB; Dako, Glostrup, Denmark) and counterstaining with hematoxylin.

Evaluation of immunostaining. Positive Sox 17 immunostaining was identified as a brown nuclear color and graded according to both intensity and percentage of cells with positive staining to eliminate the heterogeneous staining pattern. The evaluation of Sox 17 staining was carried out blindly by microscopic examination of the tissue sections by two observers (including one pathologist). Sox17 staining intensity was scored as $0,1+$, $2+$ and $3+$. The percentage of Sox17-positive cells was also scored into 4 categories: 1 (0-25\%), 2 (26-50\%), 3 (51-75\%), and $4(76-100 \%)$. On the basis of the immunoreactive score, the staining pattern was defined as: negative (0), weak (1-4), moderate (6-8) and strong (9-12). The optimal cutoff point for staining score was calculated using MedCalc software for Windows, version 12.5 (MedCalc Software, Ostend, Belgium). The best area under the ROC curves (AUC) was used to determine the optimal cutoff point of staining. Based on the optimal value of the cutoff points for the Sox 17 scores that was identified as 4 , we grouped negative and weak staining as low expression and moderate and strong staining as high 
A


B

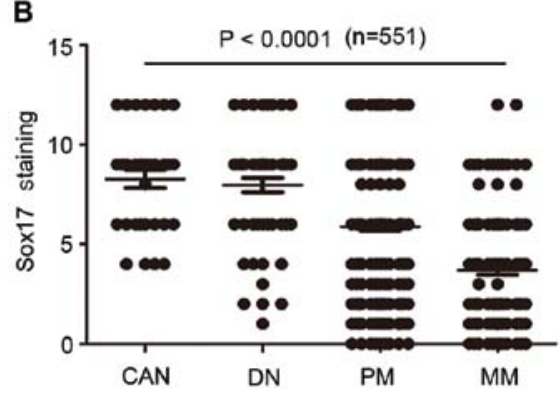

DN
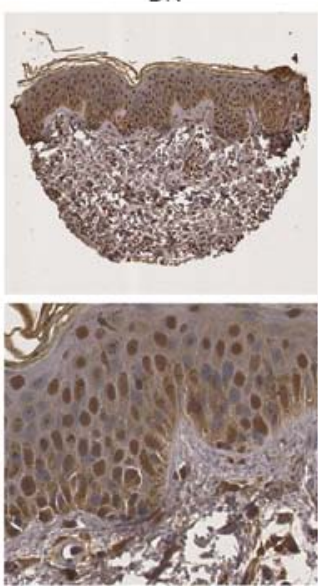
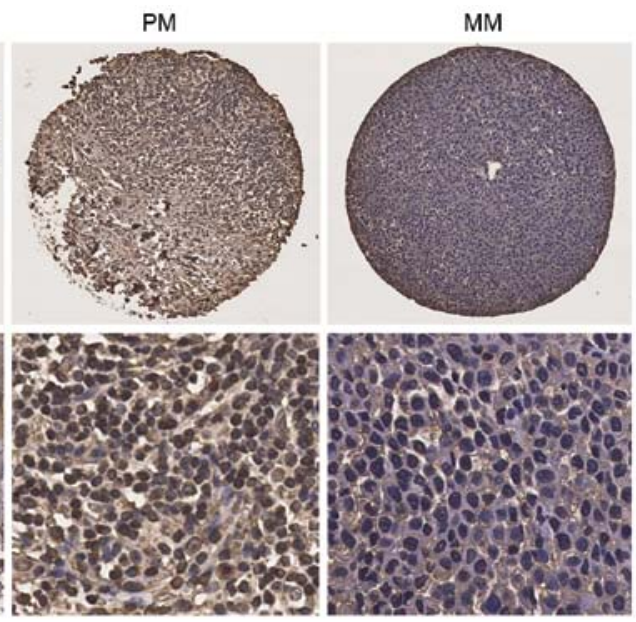

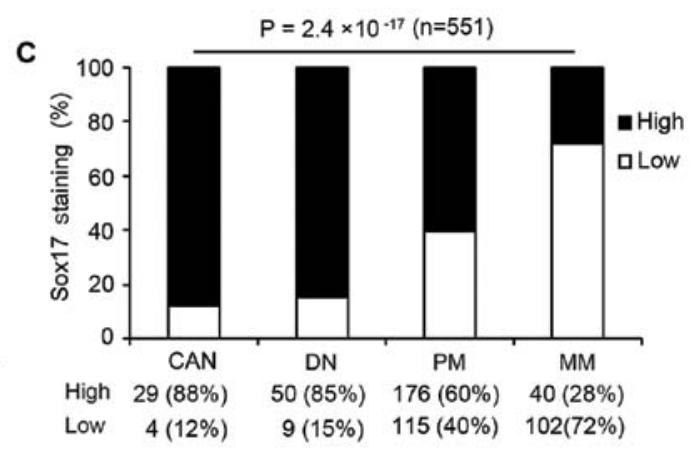

Figure 2. Reduced Sox 17 expression inversely correlates with melanoma progression. (A) Representative images of CAN with strong Sox 17 staining, DN with moderate Sox 17 staining, PM with weak Sox 17 staining and MM with negative Sox 17 staining (upper panel: scale bar, $40 \mu \mathrm{m}$; lower panel: scale bar, $20 \mu \mathrm{m}$ ). (B) Kruskal-Wallis test for differences in Sox17 staining among CAN, DN, PM and MM. The mean is depicted as a horizontal line in each group (n=525; $\mathrm{P}<0.0001)$. (C) High Sox 17 staining was decreased from CAN to DN, PM and MM ( $\mathrm{n}=525 ; \mathrm{P}=2.4 \times 10^{-17}, \chi^{2}$ test). Sox17, SRY-box containing gene 17; CAN, common acquired nevi; DN, dysplastic nevi, PM, primary melanoma; MM, metastatic melanoma.

expression. The correlation between Sox17 and p27 expression was studied in 374 melanoma samples which were common to the present study and the previously published study on the prognostic significance of p27 expression in melanoma (22).

Statistical analysis. Differences in the patient demographic and clinical characteristics and Sox17 expression were evaluated by Kruskal-Wallis test and Chi-square $\left(\chi^{2}\right)$ test between patient subgroups. Survival time was calculated from the date of melanoma diagnosis to the date of death or last follow-up. The effect of Sox17 expression on overall and disease-specific survival was evaluated by Kaplan-Meier analysis and log-rank test. Univariate and multivariate Cox proportional hazard regression models were performed to estimate the hazard ratios (HRs) or adjusted HRs and their $95 \%$ confidential intervals (CIs). A P-value of $<0.05$ was considered to indicate a statistically significant result. SPSS version 16 (SPSS Inc., Chicago, IL, USA) software was used for all analyses.

\section{Results}

Sox17 expression is inversely correlated with melanoma progression. Sox 17 staining was stronger in common acquired nevi and dysplastic nevi biopsies than that in the primary and metastatic melanoma cases (Fig. 2A). Kruskal-Wallis test on the Sox17 scoring pattern in the patient samples revealed that Sox 17 expression was significantly decreased from common acquired nevi (mean 8.3) and dysplastic nevi (mean 7.9) to primary melanoma (mean 5.8) and to metastatic melanoma (mean 3.6) $(\mathrm{n}=551$; $\mathrm{P}<0.0001$; Fig. 2B). Furthermore, the Chi-square test revealed that the percentage of high Sox 17 staining was significantly reduced in primary melanoma $(60 \%)$ and metastatic melanoma $(28 \%)$ compared to the common acquired nevi (88\%) and dysplastic nevi (85\%) $(\mathrm{n}=551$; $\mathrm{P}=2.4 \times 10^{-17}$; Fig. 2C).

Sox17 expression inversely correlates with American Joint Committee on Cancer (AJCC) stage, tumor thickness and ulceration. The Kruskal-Wallis test on the Sox17 scoring pattern in the melanoma samples revealed that Sox 17 expression was significantly decreased from early stage (AJCC I and II; mean 5.9) to advanced stage (AJCC III and IV; mean 3.7) melanoma ( $\mathrm{P}<0.0001$, Fig. 3A). As shown in Fig. 3B and Table I, high expression of Sox17 was detected in $61 \%$ of melanomas at AJCC stage I and II compared to $28 \%$ of melanomas at AJCC III and IV $\left(\mathrm{P}=4.5 \times 10^{-10}\right)$, indicating that decreased Sox 17 expression may play an important role in primary to metastatic melanoma transition. Next, we found that in primary melanoma, Sox17 expression was decreased in tumors with thickness $>4.00 \mathrm{~mm}$ (mean 3.9), compared to melanomas with thickness $\leq 4.00 \mathrm{~mm}$ (mean 6.3) $(\mathrm{P}<0.0001$, Fig. 3C). Moreover, high Sox17 expression was found in $66 \%$ of melanomas with thickness $\leq 4.00 \mathrm{~mm}$, compared to $37 \%$ of tumors with thickness $>4.00 \mathrm{~mm}(\mathrm{P}=0.00004$; 
Table I. Sox 17 staining and clinicopathologic characteristics of the 433 melanoma cases.

\begin{tabular}{|c|c|c|c|c|}
\hline \multirow[b]{2}{*}{ Variables } & \multicolumn{2}{|c|}{ Sox17 staining } & \multirow[b]{2}{*}{$\begin{array}{l}\text { Total } \\
\mathrm{n}(\%)\end{array}$} & \multirow[b]{2}{*}{ P-value } \\
\hline & $\begin{array}{l}\text { Low } \\
\mathrm{n}(\%)\end{array}$ & $\begin{array}{l}\text { High } \\
\text { n (\%) }\end{array}$ & & \\
\hline \multicolumn{5}{|l|}{ All melanoma $(n=433)$} \\
\hline \multicolumn{5}{|l|}{ Age, years } \\
\hline$\leq 60$ & $112(50.5)$ & $110(49.8)$ & $222(51.3)$ & \multirow[t]{2}{*}{0.89} \\
\hline$>60$ & $105(49.5)$ & $106(50.2)$ & $211(48.7)$ & \\
\hline \multicolumn{5}{|l|}{ Gender } \\
\hline Male & $133(52.0)$ & $123(48.0)$ & $256(59.1)$ & \multirow[t]{2}{*}{0.36} \\
\hline Female & $84(47.5)$ & $93(52.5)$ & $177(40.9)$ & \\
\hline \multicolumn{5}{|l|}{ AJCC stage } \\
\hline I & $47(27.5)$ & $124(72.5)$ & $171(39.9)$ & \multirow[t]{4}{*}{$4.6 \times 10^{-15 a}$} \\
\hline II & $68(56.7)$ & $52(43.3)$ & $120(28.0)$ & \\
\hline III & $46(79.3)$ & $12(20.7)$ & $58(13.5)$ & \\
\hline IV & $53(66.3)$ & $27(33.7)$ & $80(18.6)$ & \\
\hline \multicolumn{5}{|c|}{ Primary melanoma $(n=291)$} \\
\hline \multicolumn{5}{|l|}{ Age, years } \\
\hline$\leq 60$ & $57(40.1)$ & $85(59.9)$ & $142(48.8)$ & \multirow[t]{2}{*}{0.83} \\
\hline$>60$ & $58(38.9)$ & $91(61.1)$ & $149(51.2)$ & \\
\hline \multicolumn{5}{|l|}{ Gender } \\
\hline Male & $62(39.5)$ & $95(60.5)$ & $157(54.0)$ & \multirow[t]{2}{*}{1.0} \\
\hline Female & $53(39.6)$ & $81(60.4)$ & $134(46.0)$ & \\
\hline \multicolumn{5}{|l|}{ Tumor thickness (mm) } \\
\hline$\leq 4$ & $78(33.6)$ & $154(66.4)$ & $232(79.7)$ & \multirow[t]{2}{*}{0.00004} \\
\hline$>4$ & $37(62.7)$ & $22(37.3)$ & $59(20.3)$ & \\
\hline \multicolumn{5}{|l|}{ Ulceration } \\
\hline Absent & $88(36.7)$ & $152(63.3)$ & $240(82.5)$ & \multirow[t]{2}{*}{0.03} \\
\hline Present & $27(52.9)$ & $24(47.1)$ & $51(17.5)$ & \\
\hline \multicolumn{5}{|l|}{ Subtype } \\
\hline Lentigo maligna & $18(27.7)$ & $47(72.3)$ & $65(24.3)$ & \multirow[t]{4}{*}{0.0003} \\
\hline Superficial spreading & $32(30.5)$ & $73(69.5)$ & 105 (39.2) & \\
\hline Nodular & $28(60.9)$ & $18(39.1)$ & $46(17.2)$ & \\
\hline Unspecified & $26(50.0)$ & $26(50.0)$ & $52(19.3)$ & \\
\hline \multicolumn{5}{|l|}{ Site $^{b}$} \\
\hline Sun-protected & $84(39.6)$ & $128(60.4)$ & $212(72.9)$ & \multirow[t]{2}{*}{0.95} \\
\hline Sun-exposed & $31(39.2)$ & $48(60.8)$ & $79(27.1)$ & \\
\hline \multicolumn{5}{|c|}{ Metastatic melanoma $(n=142)$} \\
\hline \multicolumn{5}{|l|}{ Age, years } \\
\hline$\leq 60$ & $55(68.8)$ & $25(31.2)$ & $80(56.3)$ & 0.35 \\
\hline$>60$ & $47(75.8)$ & $15(24.2)$ & $62(43.7)$ & \\
\hline Gender & & & & \\
\hline Male & $71(71.7)$ & $28(28.3)$ & 99 (69.7) & 0.96 \\
\hline Female & $31(72.1)$ & $12(27.9)$ & $43(30.3)$ & \\
\hline
\end{tabular}

Sox 17, SRY-box containing gene 17; AJCC, American Joint Committee on Cancer. ${ }^{\mathrm{a} C o m p a r i s o n ~ a m o n g ~ A J C C ~ s t a g e ~ I-I V, ~} \chi^{2}$ test; ${ }^{\text {b}}$ Sun-protected sites: trunk, arm, leg and feet; sun-exposed sites: head and neck.

Fig. 3D and Table I). Furthermore, Sox17 expression decreased in the melanomas with ulceration (mean 4.6), compared to the melanomas with no ulceration (mean 6.1) $(\mathrm{P}=0.003$;
Fig. 3E), which is consistent with the Chi-square test results that high Sox17 expression was found in $47 \%$ of melanomas with ulceration, compared to $63 \%$ of melanomas with no 
A
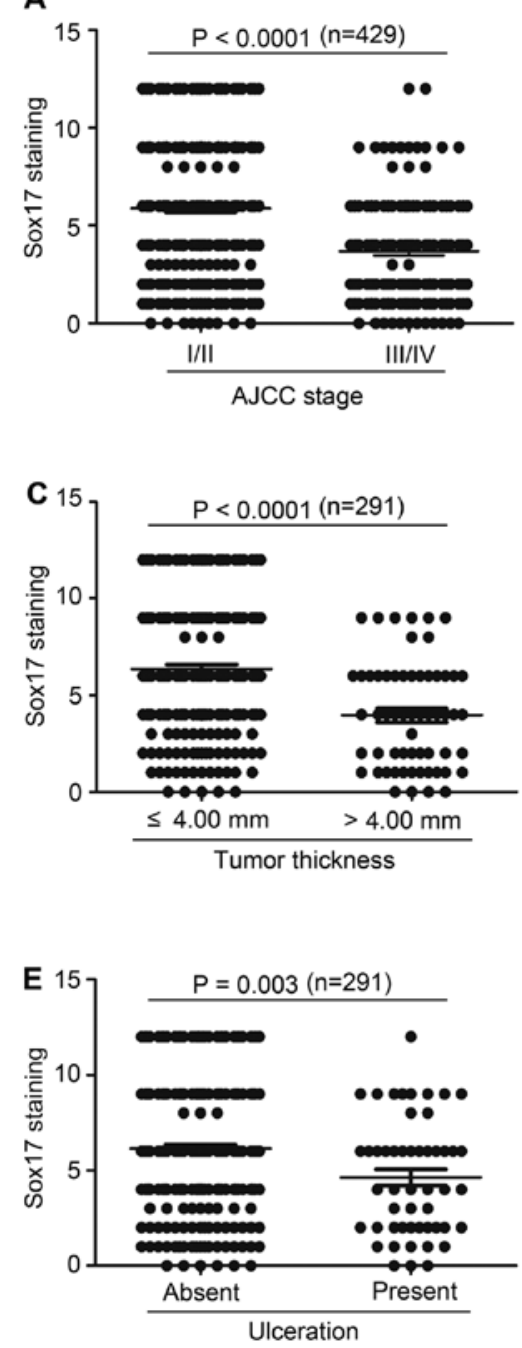


Figure 3. Sox 17 expression inversely correlates with melanoma AJCC stage, tumor thickness and ulceration. (A) Kruskal-Wallis test for differences in Sox17 staining between AJCC stage I/II to stage III/IV. The mean is depicted as a horizontal line in each group ( $\mathrm{n}=429 ; \mathrm{P}<0.0001)$. (B) High Sox17 expression was decreased from AJCC stage I/II to III/IV ( $\mathrm{n}=429 ; \mathrm{P}=4.5 \times 10^{-10}, \chi^{2}$ test). (C) The differences in Sox17 staining between melanoma thickness $\leq 4.00 \mathrm{~mm}$ and $>4.00 \mathrm{~mm}$. The mean is depicted as a horizontal line in each group ( $\mathrm{n}=291 ; \mathrm{P}<0.0001$, t-test). (D) Melanomas $>4.00 \mathrm{~mm}$ had a lower percentage of high Sox17 expression compared with melanomas $\leq 4.00 \mathrm{~mm}\left(\mathrm{n}=291 ; \mathrm{P}=0.00004, \chi^{2}\right.$ test). (E) The differences in Sox 17 staining between melanoma patients with no ulceration and with ulceration. The mean is depicted as a horizontal line in each group ( $\mathrm{n}=291 ; \mathrm{P}=0.003$, t-test). (F) Decreased Sox 17 expression is correlated with ulceration of melanomas $\left(\mathrm{n}=291 ; \mathrm{P}=0.03, \chi^{2}\right.$ test). Sox 17, SRY-box containing gene 17; AJCC; American Joint Committee on Cancer.

ulceration ( $\mathrm{P}=0.03$; Fig. 3F and Table I). Moreover, Sox17 expression was significantly different among the different subtypes of melanoma $(\mathrm{P}=0.00003$; Table I). We did not find significant correlations between Sox 17 expression and other variables (Table I).

Reduced Sox17 expression is associated with poor melanoma patient survival. In the 413 melanoma patients, Kaplan-Meier survival curve revealed that the patients with high Sox17 expression had a better overall and disease-specific 5-year survival than patients with low Sox17 expression $(\mathrm{P}=0.005$ and 0.004, respectively; Fig. 4A). More meaningful, we further investigated whether Sox17 expression was associated with melanoma patient 10 -year survival. We analyzed the 267 melanoma patients and found that the patients with high Sox 17 expression also had a better overall and disease-specific 10-year survival than patients with low Sox17 expression $(\mathrm{P}=0.038$ and 0.046 , respectively; Fig. 4B).
Sox17 expression is an independent prognostic marker for melanoma. To further estimate the prognostic value of Sox17 expression in melanoma patients, univariate and multivariate Cox proportional hazard analyses were performed. When analyzed using univariate Cox regressive analysis, patients with high Sox17 expression had significantly better overall survival (HR, 0.65; 95\% CI, 0.48-0.89; $\mathrm{P}=0.006)$ and disease-specific (HR, 0.63; 95\% CI, 0.45-0.87; $\mathrm{P}=0.005$; Table II) 5-year survival among the 413 melanoma patients. Concerning 10-year survival, univariate Cox regressive analysis further confirmed that patients with high Sox17 expression had significantly better overall survival (HR, 1.48; 95\% CI, 1.02-2.14; $\mathrm{P}=0.04)$ and disease-specific (HR, 1.51; 95\% CI, 1.00-2.28; $\mathrm{P}=0.05$; Table II) 10 -year survival in the 267 melanoma patients.

Furthermore, multivariate Cox regressive analysis was carried out with age, gender and Sox17 expression together as the variables. The results indicated that Sox17 expression 
Table II. Univariate and multivariate Cox regression analyses of the 5- or 10-year survival of the melanoma patients.

\begin{tabular}{|c|c|c|c|c|c|c|c|c|c|c|}
\hline \multirow[b]{2}{*}{ Variable } & \multicolumn{5}{|c|}{ Univariate Cox regression } & \multicolumn{5}{|c|}{ Multivariate Cox regression } \\
\hline & $\beta^{\mathrm{a}}$ & SE & HR & $95 \% \mathrm{CI}$ & $\mathrm{P}$-value & $\beta^{\mathrm{a}}$ & SE & HR & $95 \% \mathrm{CI}$ & P-value \\
\hline \multicolumn{11}{|c|}{ 5-year overall survival $(n=413)$} \\
\hline Age & -0.17 & 0.15 & 0.85 & $0.63-1.14$ & 0.27 & -0.16 & 0.15 & 0.85 & $0.63-1.15$ & 0.28 \\
\hline Gender & 0.07 & 0.08 & 1.07 & $0.92-1.25$ & 0.39 & -0.12 & 0.16 & 1.12 & $0.83-1.53$ & 0.46 \\
\hline Sox 17 & -0.42 & 0.15 & 0.65 & $0.48-0.89$ & 0.006 & 0.42 & 0.15 & 1.52 & $1.13-2.06$ & 0.006 \\
\hline \multicolumn{11}{|c|}{5 -year disease-specific survival $(n=413)$} \\
\hline Age & -0.05 & 0.16 & 0.95 & $0.69-1.31$ & 0.76 & -0.05 & 0.16 & 0.95 & $0.69-1.31$ & 0.77 \\
\hline Gender & 0.06 & 0.08 & 1.06 & $0.90-1.25$ & 0.47 & 0.10 & 0.17 & 1.11 & $0.80-1.54$ & 0.54 \\
\hline Sox 17 & -0.47 & 0.17 & 0.63 & $0.45-0.87$ & 0.005 & 0.46 & 0.17 & 1.59 & $1.15-2.20$ & 0.005 \\
\hline \multicolumn{11}{|c|}{10 -year overall survival $(n=267)$} \\
\hline Age & -0.78 & 0.20 & 0.46 & $0.31-0.68$ & $7 \times 10^{-5}$ & -0.78 & 0.20 & 0.46 & $0.31-0.68$ & $8 \times 10^{-5}$ \\
\hline Gender & 0.17 & 0.19 & 1.19 & $0.82-1.73$ & 0.37 & 0.11 & 0.19 & 1.12 & $0.77-1.63$ & 0.56 \\
\hline Sox 17 & 0.39 & 0.19 & 1.48 & $1.02-2.14$ & 0.04 & 0.41 & 0.19 & 1.51 & $1.04-2.19$ & 0.03 \\
\hline \multicolumn{11}{|c|}{ 10-year disease-specific survival $(\mathrm{n}=267)$} \\
\hline Age & -0.48 & 0.21 & 0.62 & $0.41-0.94$ & 0.02 & -0.47 & 0.21 & 0.63 & $0.41-0.95$ & 0.03 \\
\hline Gender & 0.24 & 0.21 & 1.27 & $0.84-1.93$ & 0.25 & 0.21 & 0.21 & 1.23 & $0.81-1.87$ & 0.33 \\
\hline Sox 17 & 0.42 & 0.21 & 1.51 & $1.00-2.28$ & 0.05 & 0.43 & 0.21 & 1.54 & $1.02-2.32$ & 0.04 \\
\hline
\end{tabular}

${ }^{a} \beta$, regression coefficient. Coding of variables was as follows: age was coded as 1 ( $\leq 60$ years) and 2 ( $>60$ years). Gender was coded as 1 (male) and 2 (female). Low Sox 17 staining was coded as 1 and high Sox 17 staining was coded as 2 . SE, standard error of $\beta$; HR, hazard ratio; CI, confidence interval; Sox17, SRY-box containing gene 17.

$\mathbf{A}$

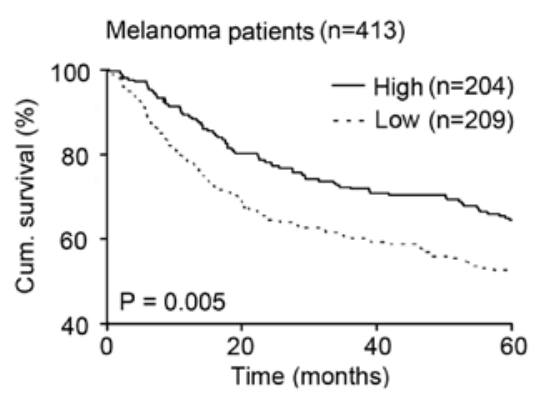

B

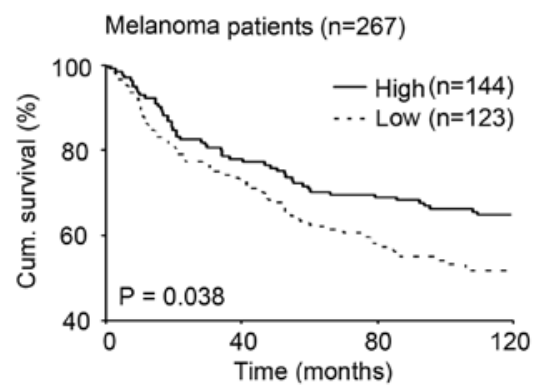

Disease-specific 5-y survival

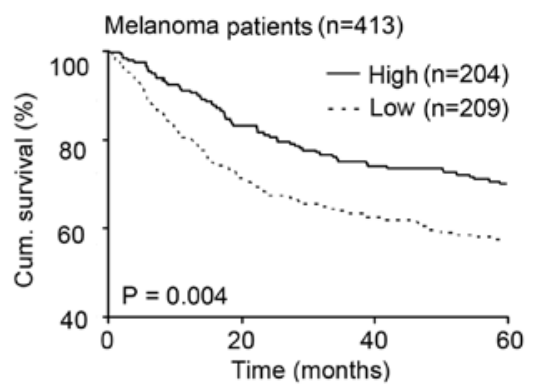

Disease-specific 10-y survival

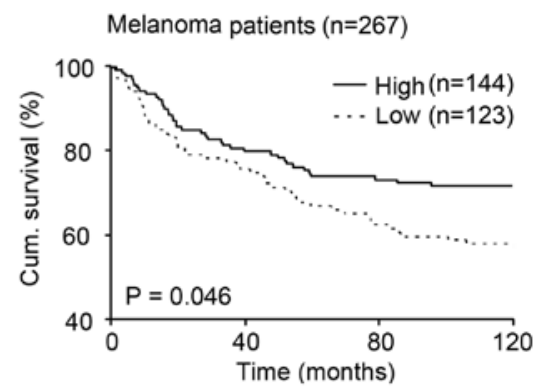

Figure 4. Kaplan-Meier analysis of the correlation between Sox 17 expression and 5- and 10-year melanoma patient survival. (A) Five-year overall and diseasespecific survival of the melanoma patients ( $\mathrm{n}=413 ; \mathrm{P}=0.005$ and 0.004 , respectively, log-rank test); (B) 10 -year overall and disease-specific survival of the melanoma patients ( $\mathrm{n}=267 ; \mathrm{P}=0.038$ and 0.046 , respectively, log-rank test).

is an independent prognostic factor for both overall (HR, $1.59 ; 95 \% \mathrm{CI}, 1.15-2.20 ; \mathrm{P}=0.005$; Table II) 5-year survival 1.52; 95\% CI, 1.13-2.06; $\mathrm{P}=0.006)$ and disease-specific (HR, in the 413 melanoma patients. Concerning 10-year survival, 
A
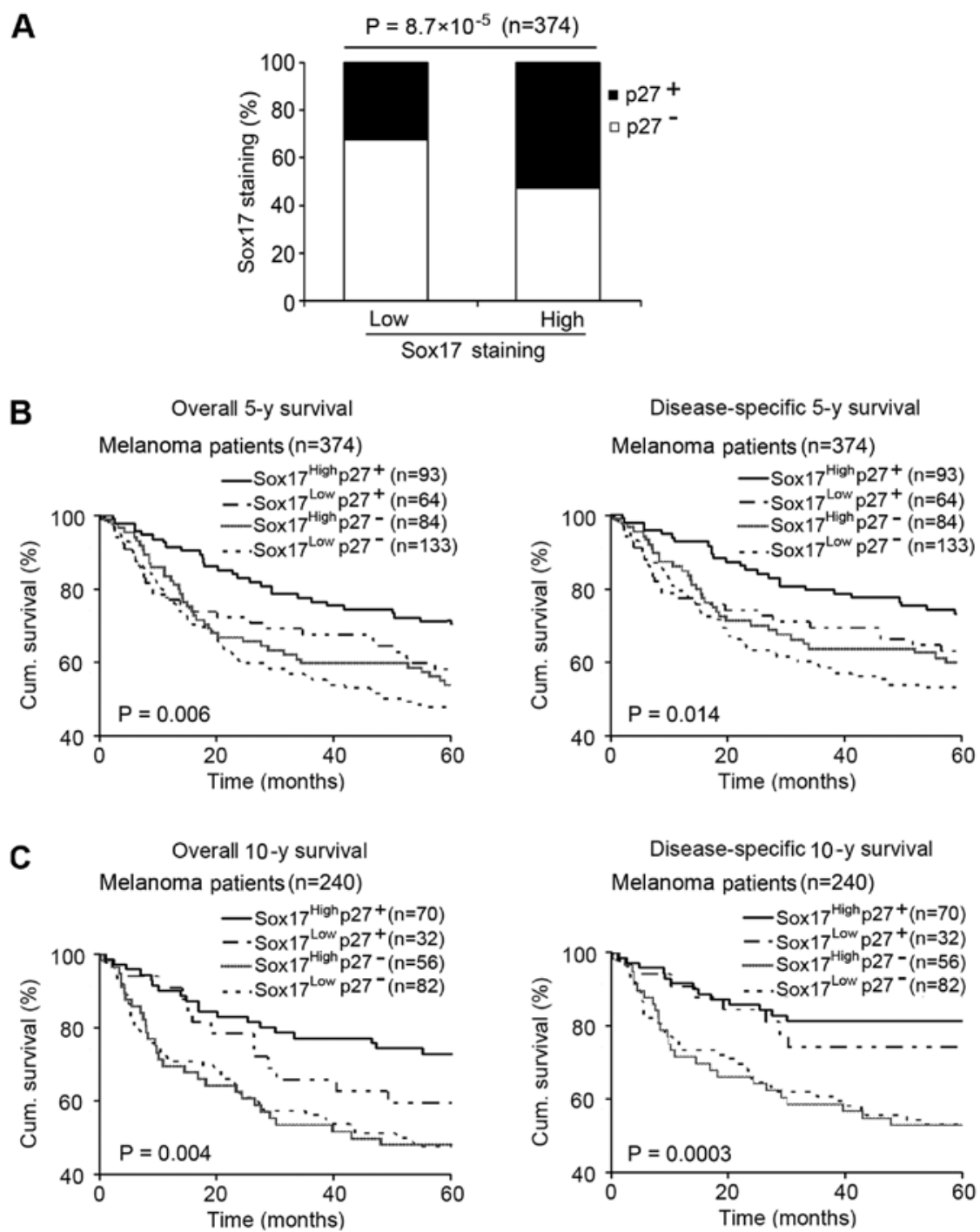

Figure 5. Sox 17 expression positively correlates with p27 expression and the influence of their concomitant expression on melanoma patient survival. (A) Positive correlation between Sox 17 and p27 expression in melanoma. Melanomas which had high Sox 17 expression also had a significantly higher percentage of positive p27 staining ( $\mathrm{n}=374 ; \mathrm{P}=8.7 \times 10^{-5}, \chi^{2}$ test) (+, positive; -, negative). (B) Kaplan-Meier analysis of the correlation between combined Sox17 and p27 expression and overall or disease-specific 5-year survival of the melanoma patients ( $\mathrm{n}=374 ; \mathrm{P}=0.006$ and 0.014 , respectively, log-rank test). (C) Kaplan-Meier analysis of the correlation between combined Sox17 and p27 expression and overall or disease-specific 10-year survival of the melanoma patients $(n=240 ; P=0.004$ and 0.0003, respectively, log-rank test). Sox17, SRY-box containing gene 17.

multivariate Cox regressive analysis further demonstrated that Sox17 expression was also correlated with both overall (HR, $1.51 ; 95 \% \mathrm{CI}, 1.04-2.19 ; \mathrm{P}=0.03)$ and disease specific (HR, 1.54 ; $95 \%$ CI, 1.02-2.32; $\mathrm{P}=0.04$; Table II) 10 -year survival in the 267 melanoma patients.

Sox 17 expression positively correlates with p27 expression, and their concomitant expression influences melanoma patient survival. Reduced p27 expression has been shown to be associated with increased invasion and unfavorable patient survival $(20,21)$. Since the TMA we used for Sox17 staining in this study was the same as that previously stained for p27 (22), we were able to analyze the correlation between Sox17 and p27 expression. As shown in Fig. 5A, high Sox 17 was positively correlated with positive p27 expression $\left(\mathrm{P}=8.7 \times 10^{-5}\right)$. We then analyzed the effect of combined Sox 17 and p27 expression on patient survival and found that patients with both high Sox17 and positive p27 expression showed a significantly increased 5-year overall and disease-specific survival, compared with the patients with other expression patterns of Sox17 and p27 using Kaplan-Meier survival curves $(\mathrm{P}=0.006$ and 0.014 , respectively; Fig. 5B). More meaningful, concerning 10-year survival, the analysis also demonstrated that a high Sox17 and positive p27 expression pattern exerted a more significant influence on melanoma patient survival $(\mathrm{P}=0.004$ and 0.0003 , respectively; Fig. 5C).

\section{Discussion}

A number of studies have demonstrated that Sox17 expression is downregulated in different types of human cancers and it appears to act as a tumor suppressor (17-19). However, a recent report demonstrated that Sox 17 expressed in tumor endothelial cells promoted tumor angiogenesis and vascular destabilization by upregulation of VEGFR2 (26). To understand the role of Sox 17 in melanoma progression, we used TMA technology 
and immunohistochemistry to investigate Sox17 expression in 525 cases of pigmented skin lesions at different stages.

Our results revealed that Sox17 expression was markedly decreased in primary melanoma when compared with the expression in common acquired nevi and dysplastic nevi and was further downregulated in metastatic melanoma compared with primary melanoma. This indicates that reduced Sox 17 activity may have a relationship with the transformation from benign neoplasia to malignancy, as well as in tumor progression from primary to metastatic melanoma. This finding is consistent with previous research that found that Sox17 was rarely detected by immunohistochemistry in gastric and colon cancers, whereas strong nuclear staining of Sox 17 was found in $70 \%$ of benign gastric and intestinal tumors (19). It is therefore conceivable that Sox 17 protects benign tumors from malignant progression at an early stage of tumorigenesis, and downregulation of Sox17 contributes to malignant progression.

It was also reported that Sox 17 methylation was found in $60.2 \%$ of primary human lung cancer samples. The promoter region methylation silenced the expression of Sox17, and re-expression of Sox17 inhibited Wnt signaling in lung cancer cells (27). The global analysis of CpG island hypermethylation and gene expression in colorectal cancer cell lines has revealed that Sox 17 gene silencing is associated with DNA hypermethylation (18). Recent research demonstrated that loss of Sox17 expression is correlated to promoter region hypermethylation in esophageal cancer. Restoration of Sox 17 expression suppresses TCF/ $\beta$-catenin-dependent transcription and colony formation (28). Furthermore, Sox 17 expression is often epigenetically silenced by DNA methylation in early gastric cancer and the introduction of Sox17 into cancer cells suppresses cancer cell growth (29).

In addition, we found that Sox 17 expression was inversely correlated with AJCC stage, ulceration and tumor thickness ( $\leq 4.00 \mathrm{vs} .>4.00 \mathrm{~mm}$ ) of melanomas. A reflection of the intense metastatic propensity of melanomas is the fact that the metastatic potential is measured on a scale of millimeters, where a tumor thickness of $4.00 \mathrm{~mm}$ predicts a high risk of cancer dissemination and death (30). By constructing Kaplan-Meier survival curves, we found that reduced Sox17 was associated with the poor survival of melanoma patients and this correlation was further confirmed by univariate Cox regression analyses. Furthermore, after adjustment for age and gender in multivariate Cox regression models, the analysis further indicated that Sox17 expression is an independent prognostic marker for melanoma patients. To our knowledge, this is the first study to reveal the prognostic value of Sox17 expression in tumor patients by TMA technology.

p27, a cell-cycle inhibitory molecule, inhibits the catalytic activity of CDK4. Increased levels of the p27 protein typically cause cells to arrest in the G1 phase of the cell cycle (20). It has been reported that Sox 17 overexpression in human colon cancer cell lines suppressed the hyperactive $\beta$-catenin activity as well as reduced cyclin D1 expression and repressed cell proliferation $(18,31)$. Colony formation assays revealed that Sox17 suppressed lung cancer cell proliferation (27). Sox17 knockdown in gastric cancer cells enhanced cyclin D1 expression levels and reduced p27 expression levels (12). The expression of Sox 17 and p27 increased in parallel before myelin basic protein expression in oligodendrocytes (32). In addition, it has been demonstrated that reduced nuclear p27 expression is associated with a worse cancer patient survival (20-22).

Here, our results revealed that in 374 melanoma samples, melanomas which had high Sox17 expression also had a significantly higher percentage of positive p27 staining. Notably, patients with both high Sox 17 and positive p27 expression had an extended 5- and 10-year overall and disease-specific survival as compared to patients with both low Sox17 and negative p27 expression. Based on this, we speculate that Sox 17 may be a positive regulator of $\mathrm{p} 27$ and therefore, reduced Sox 17 expression has an inhibitory effect on melanoma progression.

In conclusion, this study shows that Sox 17 expression is significantly decreased in association with progression of human melanoma. Strikingly, reduced Sox 17 expression correlates with a worse 5- and 10-year survival of melanoma patients and is an independent prognostic factor for melanoma patients. Moreover, there is a significant positive correlation between Sox17 and p27 expression in melanoma biopsies, and their concomitant reduced expression is inversely correlated with melanoma patient survival. These data suggest that Sox17 plays an important role in melanoma pathogenesis and it may serve as a promising prognostic marker for melanoma patients.

\section{Acknowledgements}

We acknowledge Dr Guangdi Chen for his previous research on p27. This study was supported by the Canadian Institutes of Health Research (CCI-117958), the Cancer Research Society and the Canadian Dermatology Foundation. Dr Jing Lu is supported by the National Natural Science Foundation of China (81101731).

\section{References}

1. Miller AJ and Mihm MC Jr: Melanoma. N Engl J Med 355: 51-65, 2006.

2. Trinh VA: Current management of metastatic melanoma. Am J Health Syst Pharm 65: S3-S8, 2008.

3. Geller AC, Swetter SM, Brooks K, Demierre MF and Yaroch AL: Screening, early detection, and trends for melanoma: current status (2000-2006) and future directions. J Am Acad Dermatol 57: 555-576, 2007.

4. Geller AC, Swetter SM, Oliveria S, Dusza S and Halpern AC: Reducing mortality in individuals at high risk for advanced melanoma through education and screening. J Am Acad Dermatol 65: S87-S94, 2011.

5. Turner RM, Bell KJ, Morton RL, et al: Optimizing the frequency of follow-up visits for patients treated for localized primary cutaneous melanoma. J Clin Oncol 29: 4641-4646, 2011.

6. Gubbay J, Collignon J, Koopman P, et al: A gene mapping to the sex-determining region of the mouse $\mathrm{Y}$ chromosome is a member of a novel family of embryonically expressed genes. Nature 346: 245-250, 1990.

7. Wegner M: From head to toes: the multiple facets of Sox proteins Nucleic Acids Res 27: 1409-1420, 1999.

8. Burtscher I, Barkey W, Schwarzfischer M, Theis FJ and Lickert H: The Sox17-mCherry fusion mouse line allows visualization of endoderm and vascular endothelial development. Genesis 50: 496-505, 2012.

9. Niakan KK, Ji H, Maehr R, et al: Sox17 promotes differentiation in mouse embryonic stem cells by directly regulating extraembryonic gene expression and indirectly antagonizing self-renewal. Genes Dev 24: 312-326, 2010.

10. Chen HL, Chew LJ, Packer RJ and Gallo V: Modulation of the Wnt/beta-catenin pathway in human oligodendroglioma cells by Sox 17 regulates proliferation and differentiation. Cancer Lett 335: 361-371, 2013. 
11. Jia Y, Yang Y, Liu S, Herman JG, Lu F and Guo M: SOX17 antagonizes WNT/ $\beta$-catenin signaling pathway in hepatocellular carcinoma. Epigenetics 5: 743-749, 2010.

12. Ye YW, Wu JH, Wang CM, et al: Sox17 regulates proliferation and cell cycle during gastric cancer progression. Cancer Lett 307 124-131, 2011.

13. LiD,Beisswenger C,HerrC, etal: Myeloid cellRelA/p65 promotes lung cancer proliferation through $\mathrm{Wnt} / \beta$-catenin signaling in murine and human tumor cells. Oncogene 33: 1239-1248, 2013.

14. Yang L, Chen Y, Cui T, et al: Desmoplakin acts as a tumor suppressor by inhibition of the Wnt/ $\beta$-catenin signaling pathway in human lung cancer. Carcinogenesis 33: 1863-1870, 2012.

15. Zeitlin BD, Ellis LM and Nor JE: Inhibition of vascular endothelial growth factor receptor-1/Wnt/\{beta\}-catenin crosstalk leads to tumor cell death. Clin Cancer Res 15: 7453-7455, 2009.

16 . Fodde $\mathrm{R}$ and Brabletz $\mathrm{T}$ : Wnt $/ \beta$-catenin signaling in cancer stemness and malignant behavior. Curr Opin Cell Biol 19: 150-158, 2007.

17. Fu DY, Wang ZMM, LiC, et al: Sox17, the canonical Wnt antagonist, is epigenetically inactivated by promoter methylation in human breast cancer. Breast Cancer Res Treat 119: 601-612, 2010.

18. Zhang W, Glockner SC, Guo M, et al: Epigenetic inactivation of the canonical Wnt antagonist SRY-box containing gene 17 in colorectal cancer. Cancer Res 68: 2764-2772, 2008.

19. Du YC, Oshima H, Oguma K, et al: Induction and down-regulation of Sox 17 and its possible roles during the course of gastrointestinal tumorigenesis. Gastroenterology 137: 1346-1357, 2009.

20. Chu IM, Hengst L and Slingerland JM: The Cdk inhibitor p27 in human cancer: prognostic potential and relevance to anticancer therapy. Nat Rev Cancer 8: 253-267, 2008.

21. Wander SA, Zhao D and Slingerland JM: p27: a barometer of signaling deregulation and potential predictor of response to targeted therapies. Clin Cancer Res 17: 12-18, 2011.
22. Chen G, Cheng Y, Zhang Z, Martinka M and Li G: Prognostic significance of cytoplasmic p27 expression in human melanoma. Cancer Epidemiol Biomarkers Prev 20: 2212-2221, 2011.

23. Tao GZ, Lehwald N, Jang KY, et al: Wnt/ $\beta$-catenin signaling protects mouse liver against oxidative stress-induced apoptosis through the inhibition of forkhead transcription factor FoxO3. J Biol Chem 288: 17214-17224, 2013.

24. Dai DL, Martinka M and Li G: Prognostic significance of activated Akt expression in melanoma: a clinicopathologic study of 292 cases. J Clin Oncol 23: 1473-1482, 2005.

25. Lin H, Wong RP, Martinka M and Li G: Loss of SNF5 expression correlates with poor patient survival in melanoma. Clin Cancer Res 15: 6404-6411, 2009.

26. Yang $\mathrm{H}$, Lee $\mathrm{S}, \mathrm{Kim} \mathrm{K}$, et al: Sox 17 promotes tumor angiogenesis and destabilizes tumor vessels in mice. J Clin Invest 123: 418-431, 2013.

27. Yin D, Jia Y, Yu Y, et al: SOX17 methylation inhibits its antagonism of Wnt signaling pathway in lung cancer. Discov Med 14: 33-40, 2012.

28. Jia Y, Yang Y, Zhan Q, et al: Inhibition of SOX17 by microRNA 141 and methylation activates the WNT signaling pathway in esophageal cancer. J Mol Diagn 14: 577-585, 2012.

29. Oishi Y, Watanabe Y, Yoshida Y, et al: Hypermethylation of Sox17 gene is useful as a molecular diagnostic application in early gastric cancer. Tumour Biol 33: 383-393, 2012.

30. Tarhini AA and Agarwala SS: Cutaneous melanoma: available therapy for metastatic disease. Dermatol Ther 19: 19-25, 2006.

31. Sinner D, Kordich JJ, Spence JR, et al: Sox 17 and Sox4 differentially regulate $\beta$-catenin/T-cell factor activity and proliferation of colon carcinoma cells. Mol Cell Biol 27: 7802-7815, 2007.

32. Sohn J, Natale J, Chew LJ, et al: Identification of Sox17 as a transcription factor that regulates oligodendrocyte development. J Neurosci 26: 9722-9735, 2006. 